\title{
LA REGLA HARTIANA DE RECONOCIMIENTO EN EL PENSAMIENTO GARZONIANO
}

\author{
Miguel Álvarez Ortega \\ Universidad de Sevilla
}

RESUMEN. El propósito de este trabajo es describir el acercamiento de Garzón Valdés a la regla hartiana de reconocimiento. Siendo uno de los primeros estudiosos en acercarse a los trabajos de Hart, el posicionamiento de Garzón no puede considerarse aislado ni estático. Existe una continua referencia a las tesis hartianas en la producción iusteórica de Garzón (especialmente una vez que se abandona la influencia de Kelsen en los setenta) que supone una revalorización progresiva. Trataré de mostrar en qué medida los trabajos de Garzón son un ejemplo de que resulta más útil para la Teoría del Derecho reinterpretar los instrumentos de Hart desde la conexión Derecho-Moral que descartarlos por completo.

Palabras clave: regla de reconocimiento, Herbert Hart, Ernesto Garzón Valdés, conexión Derecho-Moral.

ABSTRACT. The aim of this work is to describe Garzón Valdés's approach to the hartian rule of recognition. Being one of the first Spanish -speaking scholars to have commented on Hart's works, his position cannot be regarded as either isolated or static. There is a continuous reference to hartian theses within Garzón's legal theoretical production (especially once Kelsen's influence is abandoned in the seventies) which represents a progressive revalorisation. I shall try to show to which extent Garzón's works are an example that it is more useful for Legal Theory to reinterpret Hart's instruments from the Law-Morals connection thesis rather than totally discard them.

Keywords: rule of recognition, Herbert Hart, Ernesto Garzón Valdés, Law-Morals connection. 


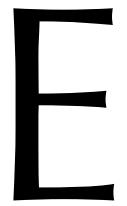

n el seno de la primera producción científica garzoniana, enfocada principalmente hacia temas de teoría del Derecho, quisiera centrarme en lo que puede considerarse grosso modo una subetapa signada por el abandono de las panvisiones jurídicas tras la publicación de su monografía sobre la Natur der Sache, a favor de un acercamiento problematista y de metodología analítica. En concreto, intentaré llevar a cabo un breve comentario sobre las diversas etapas del tratamiento otorgado por GARZÓN VALDÉS a un concepto clave del positivismo analítico anglosajón: la «regla de reconocimiento» (rule of recognition) desarrollada por Herbert HART principalmente en su obra El Concepto del Derecho (1961) ${ }^{1}$.

De manera muy sucinta, puede decirse que la regla de reconocimiento es el instrumento que idea HART para dar respuesta al problema de la validez normativa o, si se quiere, de identificación de las fuentes del Derecho, de forma que:

To say that a given rule is valid is to recognize as passing all the tests provided by the rule of recognition and so as a rule of the system. We can indeed simply say that the statement that a particular rule is valid means that it satisfies all the criteria provided by the rule of recognition ${ }^{2}$.

Esta regla es considerada como una práctica público-jurisdiccional cuya existencia es una cuestión meramente fáctica ${ }^{3}$. Los enunciados de validez suelen ser además enunciados internos, en el sentido de implicar una aceptación normativa por parte del enunciante (asunción de la norma y de la obligación de seguimiento correspondiente) ${ }^{4}$. Como oposición, los enunciados externos son los formulados por quienes se limitan a constatar regularidades de comportamiento ${ }^{5}$. Para el caso de la regla de reconocimiento, el punto de vista interno de los funcionarios (unido al seguimiento habitual de las reglas válidas) es un requisito de la existencia del ordenamiento jurídico ${ }^{6}$.

Las primeras reflexiones de GARZÓN sobre este concepto datan de una fecha tan temprana como 1967, año en que aparece su «Validez, existencia y eficacia en H. L. A. Hart» ${ }^{7}$. Aquí se contienen, en forma embrionaria, dos ideas que serían desarrolladas con posterioridad; a saber, (i) que el punto de vista interno del sector público con respecto a la regla de reconocimiento no basta para verificar la existencia del ordenamien-

${ }^{1}$ HarT, 1994: The Concept of Law, 2. ${ }^{a}$ ed., Oxford: Clarendon Press. Existe versión castellana de Genaro CARRIÓ publicada en Abeledo-Perrot, Buenos Aires $(1963,1998)$.

${ }^{2}$ HART, 1994: p. 103.

3 HART, 1994: p. 110.

${ }^{4}$ HART, 1994: p. 103.

${ }^{5}$ HART señala que cuando una persona indica que «tal cosa es Derecho» (y no, «en Inglaterra reconocen como Derecho tal otra»), realiza un: «internal statement because it manifests the internal point of view and is naturally used by one who, accepting the rule of recognition and without stating the fact that it is accepted, applies the rule in recognizing some particular rule of the system as valid. For the word "valid" is most frequently, though not always, used, in just such internal statements, applying to a particular rule of a legal system, an unstated but accepted rule of recognition». HART, 1994: p. 103.

${ }^{6}$ «There are therefore two minimum conditions necessary and sufficient for the existence of a legal system. On the one hand, those rules of behaviour which are valid according to the system's ultimate criteria of validity must be generally obeyed, and on the other hand, its rules of recognition specifying the criteria of legal validity and its rules of change and adjudication must be effectively accepted as common public standards of official behaviour by its officials». HART, 1994: p. 116.

7 GARZÓN VALDÉs, 1967: «Acerca de las nociones de validez, eficacia y existencia en H. L. A. HART», en Notas de Filosofía del Derecho, 4, pp. 23-30, incluida en la compilación Derecho, ética, política. 
to, sino que se precisa también de la capacidad o poder para imponerlo y, ii) que la relevancia del aspecto interno puede desembocar en una suerte de teoría del reconocimiento $^{8}$.

Esta segunda idea sería perfilada y reforzada diez años después en el estudio «Algunos modelos de validez normativa» (1977) ${ }^{9}$. Si aquellos que sostienen el sistema son los que formulan enunciados internos, en el sentido de aceptación de la norma, y de ahí se deriva su obligación de seguimiento, la propuesta de HART se parecería enormemente a la teoría de reconocimiento de Rudolf LAUN, según la cual la conciencia de los destinatarios delimita la validez y obligatoriedad jurídicas ${ }^{10}$. Problemas similares fueron señalados de forma un tanto más tímida por RAZ $(1970)^{11}$ y MACCORMICK $(1981)^{12}$, dando lugar a la categoría de los «enunciados desprendidos o hermenéuticos» (detached statements) que recogen el punto de vista de quien, sin estar comprometido con las reglas, describe el hecho de que otros lo estén y que HART asume en su sus Ensayos sobre Bentham (1982) ${ }^{13}$. GARZÓN no parece considerar esta novedad técnica como solución suficiente, ya que la crítica se mantiene con posterioridad a la publicación de los citados trabajos («Las limitaciones jurídicas del soberano», 1983) ${ }^{14}$. Personalmente, opino que la intención de HART es simplemente constatar que la adhesión a la regla de reconocimiento es un prerrequisito de los enunciados de validez, lo que no implica que los destinatarios puedan decidir libérrimamente qué normas pertenecen al sistema, ni tampoco cuáles les obligan. La distinción entre el «verse obligado» y «tener una obligación» supone la existencia de una norma jurídica en el segundo caso ${ }^{15}$. Ahora bien, el problema de fondo subsistente es hasta qué punto de una mera regla jurídica puede derivar-

8 GARZÓN VALDÉs, 1993: «Acerca de las nociones de validez, eficacia y existencia en H. L. A. Hart», en Derecho, ética, política, Madrid: CEC, pp. 114-115.

9 GARZÓN VALDÉS, 1977: «Algunos modelos de validez normativa», en Revista Latinoamericana de Filosofía, III, (1); reimpreso como Cuaderno de Trabajo No. 21 del Instituto de Filosofía de Derecho de la Universidad de Maracaibo (Venezuela). Aquí se citará por su inclusión en la compilación del CEC.

${ }^{10}$ GARZÓN VALDÉS, 1993: p. 93.

${ }^{11}$ RAZ, 1970: The Concept of a Legal System. An Introduction to the Theory of a Legal System, Oxford: Clarendon Press, 49.

${ }^{12}$ MacCormick, 1981: H. L. A. Hart, London: Edward Arnold, p. 32

${ }^{13}$ HART, 1982: Essays on Bentham: studies in jurisprudence and political theory, Oxford, Clarendon Press, pp. 155 ss.

${ }_{14}$ «Las limitaciones jurídicas al soberano», en GARZÓN VALDÉs, 1993: p. 195; publicado originalmente como GARZÓN VALDÉS, E., 1981: «Acerca de las limitaciones legales del soberano», en Sistema, 43-44, pp. 43 56 y reimpreso, con algunas modificaciones, en BuLYGIN et al. (comps.), 1983: El lenguaje del Derecho. Homenaje a Genaro Carrió, Buenos Aires: Abeledo-Perrot, pp. 175-180. En este trabajo se critica igualmente el acercamiento a la limitabilidad jurídica del soberano mediante la explicación de las reformas constitucionales como traslados del punto de vista interno en sentido hartiano. Más concretamente, GARZÓN señala que el tránsito desde una soberanía continuada a una autocompresiva no resulta a priori problemática. Sería el caso de la introducción de una cláusula de intangibilidad. Pero la actuación contraria (modificación de la cláusula), sería difícilmente encuadrable en un tránsito pacífico de un punto de vista interno a otro. Provocaría la invocación del derecho de resistencia (en el caso alemán) y el calificativo de revolución dependería en gran medida de las circunstancias del caso (GARZÓN VALDÉs, 1993: pp. 194-195). No obstante, debe tenerse presente que la imposibilidad de llevar a cabo válidamente este tránsito es consecuencia lógica del modelo constitucional por el que se opte y no descalifica de por sí a la «regla de reconocimiento». El propio HART parece manifestarse en este sentido cuando sostiene que el ejercicio de la omnipotencia autocomprensiva sólo puede realizarse una vez. (HART, 1994: p. 149). Cuando se incluye una cláusula de intangibilidad, lo que se pretende es precisamente eso, que se cierre la posibilidad de una vuelta atrás sin poner en marcha un verdadero nuevo proceso constituyente.

${ }^{15}$ Cfr. BAYLES, 1992: Hart's Legal Philosophy. An examination, Dordrecht: Kluwer, pp. 48-53. 
se directamente un deber de obediencia si se mantiene, como insistía HART, la separación tajante entre el Derecho y la moral.

Un primer paso hacia la superación del deslinde mencionado lo hallamos en el estudio de GARZÓN sobre El concepto de estabilidad de los sistemas políticos (1987) ${ }^{16}$, donde se defiende por vez primera que el punto de vista interno (aceptación), respecto a la regla de reconocimiento, implica una creencia en la legitimidad del sistema. En este trabajo, partiendo de la noción de estabilidad como mantenimiento de la identidad, se propone una precisión conceptual basada en la categoría de propiedad disposicional así como, a pesar de las críticas desarrolladas anteriormente, en la regla hartiana de reconocimiento. Así:

Un determinado sistema político es estable si y sólo si en los casos relacionados con el ejercicio institucionalizado del poder, sean normales o límite, tiene la tendencia a reaccionar de manera que los cambios son una explicación eficaz de la «regla de reconocimiento» y esta tendencia se mantiene durante un período de tiempo significativo de acuerdo con su contexto histórico y regional ${ }^{17}$.

Nótese que la propuesta viene referida al «sistema político» y no al «ordenamiento jurídico», espacio de definición original del instrumento. Que estamos ante nociones diversas resulta poco problemático de asumir. De acuerdo con Mario Losano, ordenamiento jurídico no es sino una aplicación más del análisis del Derecho como sistema (en concreto, el institucionalismo de Santi ROMANO), en el sentido de considerarlo como un conjunto de normas que posee o tiene la tendencia a poseer unidad, coherencia o plenitud ${ }^{18}$. Sistema político, en cambio, resulta en una noción sociológica de inspiración funcionalista (la impronta de PARSONS resulta decisiva) que pretende presentar un cuadro de interacción de los múltiples elementos, actores y factores que conforman el espacio público ${ }^{19}$. Cabe pues preguntarse, como han hecho ATIENZA y RUIZ MANERO, si el traslado de un ámbito a otro conlleva algún tipo de coste teórico. En concreto, haciendo referencia a los ejemplos del contexto latinoamericano que le sirven a GARZÓN de campo de pruebas para su teoría, estos autores señalan que la inclusión de elementos como el «capitalismo de estado benefactor» (caso de Costa Rica) desvirtuaría la regla de reconocimiento ${ }^{20}$. La contestación de GARZÓN es que la cláusula es una referencia al Estado del bienestar, habitual en constituciones contemporáneas actuando de guía política y estándar de validez jurídica ${ }^{21}$. La respuesta es plausible en la medida en que

16 GARZÓn VALdÉs, 1987: El concepto de estabilidad de los sistemas políticos, Madrid: CEC; reeditado en México: Fontamara, 1992.

17 GaRZÓn VALDÉs, 1987: p. 43.

${ }^{18}$ Cfr., Losano, 2002: Sistema e struttura nel diritto, vol. I., Milano: Giuffrè; BobBIO, N., 1991: Teoría general del Derecho, trad. cast. de E. Rozo AcuÑa, Madrid: Editorial Debate, pp. 165 ss, 195ss, 221 ss.

${ }_{19}$ Cfr. principalmente, PARSONS, 1968: La estructura de la acción social: estudio de teoría social con referencia a un grupo de recientes escritores europeos, Madrid: Guadarrama; PARSONS, 1984: El sistema social, versión castellana de J. Jiménez Blanco y J. Cazorla Pérez, Madrid: Alianza; Easton, 1953: The Political System: An Inquiry into the State of Political Science, New York: Alfred A. Knopf; EASTON, 1957: «An approach to the Analysis of Political Systems», en World Politics, XI, pp. 393-400; ElsTER, 2000: «Rational choice History: A Case of Excessive Ambition», en ARSP, 94, pp. 685 ss; para una definición de «sistema político» que incluya elementos de diversas tendencias teóricas, vid. AlCÁnTARA SÁEZ, 1994: Gobernabilidad, crisis y cambio, Madrid: CEC, p. 53.

20 AtienZa y Ruiz Manero, 1987: «Entrevista con Ernesto Garzón Valdés», en Doxa, 4, pp. 417-418.

21 Atienza y Ruiz Manero, 1987: p. 418. 
el elemento cuestionado permite a la regla de reconocimiento su función principal: ser el parámetro último de validez. Sin embargo, téngase en cuenta que GARZÓN incluye elementos de índole tan diversa como el apoyo a las compañías mineras, la lucha contra el protestantismo anglosajón, la venalidad de los jueces o el rechazo de los proyectos «desarrollistas» ${ }^{22}$. Resulta difícil admitir que elementos puramente sociológicos o económicos (muchos de los cuales poseen además cierto carácter coyuntural) determinen la pertenencia de las normas al sistema y den tal carácter a su contenido que su variación suponga una mutación en la regla de reconocimiento. Ello nos lleva a plantearnos que la aportación garzoniana es mucho más útil en el seno de la teoría jurídica: si el punto de vista interno a la regla de reconocimiento es una adhesión moral y añadimos, como anticipara en cierta medida FINNIS ${ }^{23}$ y más recientemente ha sabido mostrar VILAJOSANA ${ }^{24}$, que la identidad del ordenamiento (presupuesto del concepto de estabilidad) se basa en criterios de relevancia de índole material, la cuestión puede replantearse en el sentido de que la regla de reconocimiento proporciona criterios de validez y membresía referidos a un determinado núcleo axiológico-institucional. Cuando se mantenga la tendencia a poder ofrecer explicaciones eficaces de ese núcleo, el ordenamiento será estable.

Esta importantísima consecuencia vinculatoria Derecho-moral, sería profundizada en un trabajo clave de la producción garzoniana «Algo más acerca de la relación entre Derecho y Moral» $(1990)^{25}$. En sede de delimitación conceptual de la estabilidad, GARZÓN precisaba que la «legitimación», como aceptación de la regla de reconocimiento, debía ser distinguida de la mera aquiescencia, por hábito o apatía, o del seguimiento por razones prudenciales. No cualquier tipo de «posicionamiento» habría de servir, pues, para explicar la existencia del ordenamiento, para describir la complejidad de la actitud de los que sostienen el sistema.

No obstante, hay quien afirma, precisamente desde un positivismo de inspiración hartiana, que basta con constatar que la parte oficial aplica las normas válidas y que la generalidad de los destinatarios las siguen para defender la existencia del ordenamiento; de forma que se hace innecesario el pronunciamiento sobre las motivaciones ${ }^{26}$. La consideración de tal postura lleva a GARZÓN a plantear la posibilidad de concebir Estados en los que «la parte oficial tiene una percepción equivocada de las razones que motivan el comportamiento de sus integrantes y en donde todos ellos, además, rechazan internamente el sistema» (sería una suerte de país del error o Irrlandia), o en los que no existe ninguna creencia sobre las reglas básicas del sistema, es decir, en los que el cumplimiento se explica por mero hábito (Apatilandia) ${ }^{27}$. GARZÓN VALDÉS sostiene no solo que tales casos resultan de difícil constatación empírica y de gran inestabilidad

\footnotetext{
${ }^{22}$ GARZÓN VALDÉs, 1987: pp. 47, 51, 61, 67.

${ }_{23}$ FInNIS, 1973: «Revolutions and Continuity of Law», en SIMPSON (ed.) Oxford Essays in Jurisprudence: Second Series, Oxford University Press, pp. 69 ss.

${ }^{24}$ Vilajosana, 1996: «Towards a material criterion of identity of a legal order», en Rechtstheorie, 27, pp. 60 ss.

${ }_{25}$ Publicado en 1990, Doxa, 8, pp. 111-130. Citaré por su inclusión en la compilación del CEC.

${ }^{26}$ GARZÓN toma a Rolf SARTORIUS como modelo de esta postura. Cfr. SARTORIUS, 1987: «Positivism and the Foundation of Legal Authority», en GAVISON (ed.), Issues in Contemporary Legal Philosophy. The influence of H.L. A. Hart, Oxford, Clarendon Press; cit. en «Algo más acerca de la relación entre el Derecho y la moral», GARZÓN VALDÉS, 1993, p. 322.

${ }^{27}$ GARZÓN VALDÉS, 1993.
} 
estructural, sino que se presentan como difícilmente compatibles con la actitud de crítica reflexiva que en general HART atribuye al punto de vista interno ${ }^{28}$. GARZÓN avanza entonces un paso más para sostener la tesis de que: «entre Derecho y moral existe una relación conceptual, es decir, el sistema jurídico no puede existir sin la pretensión de corrección moral de su "regla de reconocimiento"» 29 .

Se defiende además la función de la intentio dogmatica que se halla presente en la labor del jurista práctico — señaladamente el juez-y según la cual las proposiciones jurídicas recogen una cierta pretensión de corrección moral que no puede obviar la concepción de justicia inserta en el orden y que es la que la parte oficial sustenta con su punto de vista interno ${ }^{30}$.

El propósito de GARZÓN no es soslayar que cierto sector oficial puede moverse por razones prudenciales o perentorias, que puede formular frente al Derecho enunciados desprendidos, noción que parece verse aquí revalorizada, sino el de señalar que esto no puede constituir una actitud generalizada; su intención no es negar juridicidad a los regímenes injustos, sino constatar la necesaria pretensión de legitimidad para determinar la existencia del ordenamiento. Se trata en definitiva de asumir que una coherente descripción del funcionamiento del Derecho pasa por aceptar su vinculación con la moral. La renuencia a admitir tal nexo sería el origen de buena parte de los problemas de la teoría hartiana, como viene señalando hace tiempo cierto sector doctrinal, que ha sostenido igualmente la necesidad de admitir la tesis de la vinculación para una lectura más coherente del filósofo de Oxford ${ }^{31}$. El empirismo de la regla de reconocimiento no basta por sí mismo para explicar la juridicidad (según el conocido problema del paso de los hechos a las normas) ${ }^{32}$, sino que precisa, como han indicado entre otros SCARPELLI y CATANIA, una adhesión valorativa ${ }^{33}$. De ahí la conclusión garzoniana de que: «quienes adoptan el punto de vista interno, sólo pueden explicar la validez última de las normas del sistema recurriendo a argumentos morales» ${ }^{34}$.

28 HART, 1994: p. 57.

29 GARZÓN VALDÉS, 1993: p. 324.

${ }^{30}$ GARZÓN VALDÉS, 1993: pp. 324-325. « [E]l juez que pronuncia una sentencia tiene la pretensión de que su fallo es correcto no sólo legalmente sino también moralmente». «Seguridad jurídica y equidad», en GARZÓN VALDÉS, E., 1993: p. 308.

${ }^{31}$ Cfr. MACCORMICK, 1978: Legal Reasoning and Legal Theory, Oxford: Clarendon Press, pp. 240-241; FuLLER, 1967: La moral del Derecho, México: Trillas; e incluso Joseph RAZ acabaría modificando su postura inicial de «ortodoxia hartiana» en RAZ, 1981: «The Purity of the Pure Theory», en Revue Internationale de Philosophie, 138, p. 455. En nuestro país, se han manifestado en este sentido, entre otros, DE PÁrAmO, 1984: H.L. A. Hart y la teoría analítica del Derecho, Madrid: CEC, pp. 103, 250-252; RAMOS PASCUA, 1989: La regla de reconocimiento en la teoría jurídica de H. L. A. Hart, Madrid: Tecnos, pp. 192, 195 [«existe una adhesión políticomoral a la regla de reconocimiento, porque, a juicio de los obligados, juega un papel decisivo en el mantenimiento del tipo de sociedad en que viven (...). [H] abrá de concluirse que por medio de la regla de reconocimiento el Derecho conecta necesariamente con valoraciones políticas y morales, y, consecuentemente, con la moral, al menos con la moral social dominante», p. 194]; PÉREZ LUÑO, 1991: «¿Qué deber? Consideraciones sobre el deber de obediencia al derecho con especial referencia a la teoría de H. L. A. Hart», en VVAA, Obligatoriedad y derecho, Oviedo: Universidad de Oviedo, p. 390.

32 «En última instancia, una teoría empírica de la norma fundamental tiene que fracasar porque no puede aprehender adecuadamente el problema propiamente dicho de toda norma fundamental, es decir, el paso del

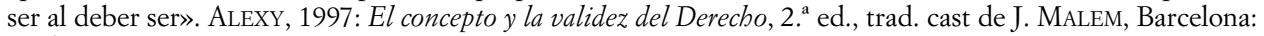
Gedisa, p. 122.

33 Cfr. SCARPElli, 1965: Cos'è il positivismo giuridico, Milano: Comunità, pp. 81 ss; CATANIA, A., 1971: «L'accettazione nel pensiero di Herbert L. A. Hart», en RIFD, 48, pp. 266-267.

${ }^{34}$ GARZÓN VALDÉS, 1993: «Seguridad jurídica y equidad». 
De esta forma, concluye el recorrido cronológico del tratamiento garzoniano de la regla de reconocimiento. Confío en que dos ideas básicas puedan extraerse de este brevísimo bosquejo. En primer lugar, que a pesar de sus imperfecciones técnicas y los importantes ataques recibidos — los más demoledores parecen seguir siendo los debidos a DWORKIN - los instrumentos hartianos pueden seguir resultando de utilidad en la ciencia jurídica. En segundo lugar, que un paso decisivo para explotar la potencialidad de estos conceptos es asumir una conexión necesaria del Derecho con la moral. Los trabajos de GARZÓN VALDÉS son un admirable ejemplo de revalorización progresiva de elementos hartianos mediante su depuración y superación desde los parámetros apuntados. 